\title{
Use of ursodeoxycholic acid in patients with hypertransaminasemia
}

\author{
Claudio Puoti, ${ }^{1}$ Maria Giuseppa Elmo ${ }^{2}$ \\ ${ }^{1}$ Department of Internal Medicine and Hepato-Gastroenterology, Marino General Hospital, Marino (RM); ${ }^{2}$ Rome-A Health \\ District, Rome, Italy
}

\begin{abstract}
Ursodeoxycholic acid (UDCA) is a hydrophilic bile acid deriving from $7 \beta$ epimerization of primary bile compound chenodeoxycholic acid. It may have antioxidant, immunomodulatory, antifibrotic and cytoprotective effects, therefore it has been extensively used in the treatment of cholestatic chronic liver diseases (primary biliary cirrhosis, primary sclerosing cholangitis, cholestasis of pregnancy, etc.). Due to the effectiveness of UDCA in decreasing serum liver enzyme levels also in non-cholestatic patients with chronic liver damage of various etiologies, this bile acid is now extensively used in clinical practice in combination with standard therapies, or as alternative treatment. In this paper, after a review of the biochemistry and physiology of UDCA, we have analyzed available data from the international literature on the efficacy and safety of UDCA in patients with hypertransaminasemia due to non-cholestatic chronic liver diseases, such as chronic viral hepatitis B and C and liver steatosis, the three main causes of aminotransferase elevation in western countries.
\end{abstract}

\section{Biosynthesis and functions of bile acids}

Bile acids (BAs), the major constituents of bile, are synthesized in the liver from cholesterol. ${ }^{1}$ Due to their biochemical characteristics, BAs allow the absorption and transport of dietary lipids and fat-soluble vitamins. ${ }^{2}$ They may be classified into primary BAs, which are synthesized in the liver, such as the cholic acid (CA) and the chenodeoxycholic acid (CDCA), and secondary bile acids, such as deoxycholic acid and lithocholic acid (LA), which are formed in the intestine through $7 \alpha$-dehydroxylation of primary bile acids by intestinal bacteria. ${ }^{3}$

More in details, BAs synthesis starts from choles-

Correspondence: Claudio Puoti, Department of Internal Medicine and Hepato-Gastroenterology, Marino General Hospital, viale XXIV Maggio, 00047 Marino (RM), Italy.

Mobile: +39.333.3744.784 - Fax: +39.06.9327.3047.

E-mail: puoti@epatologia.org

Key words: aminotransferases, bile acids, chronic viral hepatitis, steatosis, ursodeoxycholic acid.

Conflict of interests: the authors declare no potential conflict of interests.

Received for publication: 17 August 2013.

Revision received: 9 September 2013.

Accepted for publication: 21 September 2013.

This work is licensed under a Creative Commons Attribution NonCommercial 3.0 License (CC BY-NC 3.0).

CCopyright C. Puoti and M.G. Elmo, 2014

Licensee PAGEPress, Italy

Italian Journal of Medicine 2014; 8:141-147

doi:10.4081/itjm.2014.426 terol, ${ }^{4}$ following a classic or an alternative pathway. The former, which accounts for $90 \%$ of BAs synthesis, leads through a cascade of several enzymatic steps to the synthesis of $\mathrm{CA}(3 \alpha, 7 \alpha, 12 \alpha$-trihydroxy-5 $\beta$-cholanoic acid) and of CDCA ( $3 \alpha, 7 \alpha$-dihydroxy-5 $\beta$-cholanoic acid), while the latter pathway leads to the formation of CDCA only. Under normal conditions, $70 \%$ of the human pool of BAs is composed by CA and its metabolites, while $30 \%$ is represented by CDCA. ${ }^{1}$

After their synthesis, BAs are conjugated with glycine and taurine, improving their solubility. These conjugated BAs (glyco- and tauro-conjugated BAs) are present as anionic salts under physiological $\mathrm{pH}$ conditions and are therefore called bile salts. ${ }^{5}$ After glyco- or tauro-conjugation, primary BAs are excreted in the intestine, where they are de-conjugated and converted by $7 \alpha$-dehydroxylase in intestinal bacterial flora to the secondary BAs, deoxycholic acid $(3 \alpha, 12 \alpha$-dihydroxy- $5 \beta$-cholestanoic acid) and lithocholic acid (3 $\alpha$-hydroxy- $5 \beta$-cholestanoic acid). ${ }^{6}$

The intestinal conservation mechanism of bile salts is highly efficient. From $20-40 \mathrm{~g}$ of bile salts excreted daily into the bile, only $0.5 \mathrm{~g}$ are lost through fecal excretion and have to be replaced by de novo BA synthesis. This conservation is achieved through the enterohepatic circulation of bile salts, which depends on the action of several transporter proteins expressed at the basolateral and apical membrane of liver, biliary and small intestinal epithelial cells. ${ }^{6}$

In addition to their function of absorption and transport of dietary lipids and fat-soluble vitamins, BAs play an important role in decreasing cholesterol supersaturation human bile. ${ }^{7}$ It is well-known that the relative concentrations of bile salts, phospholipids, and cholesterol are the major determinants of gallstone 
formation. ${ }^{7}$ This separation was the result of an increase in the quantity of cholesterol relative to the amounts of bile salts and lecithin contained in the bile from patients with cholesterol gallstones. In other words, BAs and lecithin fully solubilize cholesterol through the formation of the so-called mixed micelles. Forty-five years ago, William Admirand and Donald Small ${ }^{8}$ plotted these three constituents simultaneously on triangular coordinates (the so-called triangle of $A d$ mirand-Small), thus achieving a complete separation of the normal and abnormal bile. This separation was the result of an increase in the quantity of cholesterol relative to the amounts of bile salts and lecithin contained in the bile from patients with cholesterol gallstones (supersaturated bile).

The enrichment of the BA pool by cholic acid did not desaturate the bile, ${ }^{7}$ on the contrary, the other primary BA (CDCA) seemed to be able to reduce cholesterol biliary supersaturation, ${ }^{9}$ although in several patients it induces an increase in serum aminotransferase levels.

An outstanding progress in the treatment of gallstone disease came from Japanese studies ${ }^{10}$ showing that ursodeoxycholic acid (UDCA), another BA, might be more effective than CDCA in dissolving gallstones with no relevant side effects. This paved the way to the use of UDCA for gallstone dissolution, which is nowadays very widely used for this purpose.

\section{Ursodeoxycholic acid for the treatment of chronic liver diseases: a fascinating history}

UDCA is a BAs derived from CDCA and a highly hydrophilic dihydroxy ( $3 \alpha, 7 \beta$-dihydroxy- $5 \beta$-cholanoic acid) bile acid. ${ }^{2,11}$ In humans, UDCA accounts for up to $4-5 \%$ of the BA pool. It is not synthesized in the liver, but it probably originates in the colon from bacterial $7 \beta$ epimerization of CDCA. ${ }^{12}$ After its formation, UDCA is passively absorbed by the colonic mucosa, thus entering the portal circulation and subsequently it enriches the pool of BAs.

BAs have both hydrophobic and hydrophilic properties that enable them to function as detergents. However, the different BAs have variable degrees of hydrophobicity and hydrophilicity, which are determined by their biochemical and physiochemical properties. LA acid is the least water soluble, whereas UDCA is much more hydrophilic. CA and CDCA acid have intermediate degrees of hydrophilicity. ${ }^{2}$ The orientation $(\alpha$ or $\beta$ ) of the hydroxyl groups relative to each other determines the bile acid hydrophilicity in these planar molecules.

Due to its high hydrophilic properties, its ability to reduce supersaturation of human bile and the lack of the hepatotoxicity of other endogenous BAs, UDCA was rapidly marketed globally for the non-surgical treatment of gallstones. Thus, millions of persons in the western countries were treated with the new drug.

After a few years, however, it was clear that UDCA did perform some other therapeutic actions, beyond its biliary litholytic properties. In 1987, Poupon et al. suggested that long-term use of UDCA was safe and effective in patients suffering from primary biliary cirrhosis (PBC). ${ }^{13}$ These preliminary results were confirmed 4 years later by a multicenter, prospective, placebo-controlled study in patients with PBC,${ }^{14}$ showing that UDCA therapy for 2-years has led to a reduction in clinically overt disease, improvement of liver blood tests and of Mayo risk score, a decrease in serum levels of immunoglobulin $M$ class anti-mitochondial autoantibodies, and of the mean histologic score in the treated group compared with placebo. Subsequent studies have shown that UDCA delays the progression rate of $\mathrm{PBC}$ resulting in a decreased need for liver transplantation..$^{15}$ Lindor et al. reported a lower mortality or need for liver transplantation in the UDCA treatment group compared with patients receiving placebo. ${ }^{16}$

These beneficial effects of UDCA were attributed to several other actions of this bile acid, such as expansion of hydrophilic pool of BAs with displacement of endogenous and more toxic BAs at hepatocyte level, competitive inhibition of the absorption of endogenous BAs at the terminal ileus, ${ }^{2}$ choleretic effects, immunomodulatory properties, cytoprotection and stabilization of liver cell structures, anti-apoptotic effects, anti-inflammatory properties. ${ }^{17}$

Several other studies did confirm the efficacy of UDCA in PBC, which is now widely used in clinical practice for the treatment of this and of other cholestatic diseases, such as primary sclerosing cholangitis and cholestatic disease of pregnancy. ${ }^{7}$

However, in 1999 , a meta-analysis ${ }^{18}$ failed to show some efficacy of UDCA in patients with PBC. More recently, a Cochrane review ${ }^{19}$ did conclude that UDCA does not demonstrate any significant benefits on mortality or liver transplantation, pruritus, or fatigue in patients with PBC. UDCA simply seemed to have a beneficial effect on serum liver enzyme levels and on histological progression compared with the control group.

Anyway, due to the apparent beneficial effects of UDCA in cholestatic liver diseases, over the past 20 years, several studies have investigated the safety and efficacy of this BA also in non-cholestatic chronic liver diseases, such as chronic viral hepatitis [hepatitis B virus (HBV)- and hepatitis C virus (HCV)-related], non-alcoholic fatty liver disease (NAFLD) and non-alcoholic steatohepatitis (NASH), etc., mainly characterized by cytolysis and an increase of serum aminotransferase levels, rather than by cholestasis. 


\section{Ursodeoxycholic acid and hypertransaminasemia: myth or fact?}

In their everyday clinical practice, physicians frequently must deal with patients showing increased aminotransferase levels, often found by chance in biochemical tests. Several causes for these enzyme alterations should be investigated, such as chronic hepatitis $\mathrm{B}$ or $\mathrm{C}(\mathrm{CHB}, \mathrm{CHC})$, liver steatosis (NAFLD, NASH), autoimmune hepatitis, alcohol abuse, hemochromatosis, drugs, etc. To achieve a correct diagnosis, several parameters should be evaluated, such as the clinical history and age of the patient, lifestyle, body mass index, risk factors, magnitude of enzymatic elevations, duration, alanine transaminase (AST)/aspartate transaminase (ALT) ratio, presence of other biochemical alterations (e.g., gamma-glutamyl transpepidase (GGT), alkaline phosphatase, bilirubin, platelet count, etc.). ${ }^{20}$

Studies on the general population in Italy have shown that approximately $17 \%$ of apparently healthy individuals have chronically elevated aminotransferase levels, often only slightly increased. In the majority of these patients the main cause is the steatosis of the liver, followed by $\mathrm{CHC}$ and $\mathrm{CHB} .{ }^{21}$

Due to the effectiveness of UDCA in decreasing serum liver enzyme levels in patients with chronic liver damage of various etiologies, this BA is now extensively used in clinical practice in combination with standard therapies or as an alternative treatment.

We intend to examine here available data on the efficacy and safety of UDCA in the 3 main causes of aminotransferase elevation in our country: $\mathrm{CHC}, \mathrm{CHB}$ and liver steatosis.

\section{Ursodeoxycholic acid and hepatitis $\mathrm{C}$ virus related chronic hepatitis}

Since the discovery of HCV in 1989 , given the lack of optimal treatment and the severe side effects of the former available therapy with recombinant interferon (IFN) plus ribavirin, ${ }^{22}$ several investigators began to evaluate the usefulness of UDCA in patients suffering from $\mathrm{HCV}$-related $\mathrm{CHC}$, non-candidates or non-responders to standard antiviral therapy. ${ }^{23}$

These studies were aimed at evaluating whether UDCA might reduce serum aminotransferase and GGT levels, decrease viral load (serum HCV RNA amounts), improve liver histology, and finally favorably modify the natural history of the disease and the progression to more severe liver damage, such as cirrhosis and hepatocellular carcinoma (HCC). ${ }^{24}$ Some trials $^{25-27}$ compared UDCA versus placebo or no intervention for CHC. The dose of UDCA ranged from 400 to $800 \mathrm{mg} /$ day, and the duration of treatment ranged from 3 to 12 months. ${ }^{24}$ Two other trials compared tauro-conjugated UDCA (TUDCA) versus placebo ${ }^{28}$ or no treatment. ${ }^{29}$ Other trials compared UDCA combined with IFN versus IFN monotherapy in patients with $\mathrm{CHC}$ for chronic hepatitis C. ${ }^{30}$

All these investigations reported a significant reduction in serum aminotransferase and GGT levels during the study ( $-14 \%$ to $-40 \%)$, and in several cases liver enzymes actually return within the normal range. However this biochemical normalization was lost at follow up, often returning to pre-treatment levels shortly after treatment. ${ }^{23}$ Furthermore, it should be underlined that this transient beneficial effects on serum liver enzymes were not seen in all patients treated with UDCA, as at least $30-40 \%$ did not show any significant biochemical improvement. ${ }^{26}$

The mechanisms of these biochemical remissions during UDCA treatment are not clearly understood. As mentioned above, it is possible that UDCA might exert some direct cytoprotective effects on the hepatocyte membrane, by replacing more toxic endogenous BAs at this level, or by modifying the hydrophobic/hydrophilic BA ratio. ${ }^{2-17}$

It still remains unclear why UDCA treatment is effective on liver biochemistry in some patients with $\mathrm{CHC}$, but ineffective in others. Some authors showed that a CDCA reduction in the hepatocytes is an important factor or cytoprotection, suggesting that UDCA exerts its cytoprotective action as a consequence of the stimulation of the efflux of cytotoxic BAs, such as CDCA from hepatocytes. ${ }^{31}$ Nojiri et al.${ }^{32}$ reported that in patients with $\mathrm{CHC}$ responding to UDCA treatment the percentage of this BA in the serum and the percentage of CDCA in biliary bile were significantly higher than in the non-responders. This might indicate that, when effective, UDCA favors a decrease of CDCA in hepatocytes, thus contributing to hepatoprotection.

Another important issue is that UDCA treatment fails to eradicate $\mathrm{HCV}$ infection, and does not decrease the amount of circulating genomes, as detected by HCV RNA serum levels. These disappointing results have been reported both in studies using only UDCA and in trials of a combined treatment with UDCA and IFN. ${ }^{24}$ It is noteworthy that even in the latter, when comparing the efficacy of antiviral therapy with IFN plus UDCA versus IFN alone, the decrease of $\mathrm{HCV}$ RNA levels and the rate of responders (i.e., HCV RNA negative patients at the end of follow up) did not differ between the two groups. This means that, despite biochemical improvement, UDCA is not able to induce an enhanced clearance of HCV RNA from serum. ${ }^{29}$

The third important question to be answered is whether UDCA treatment can improve liver histology in patients with CHC. Only a few studies actually address this issue, probably due to the difficulties to perform a second liver biopsy shortly after the pre-treatment one. In the study of Attili et al. ${ }^{33} 18 \mathrm{pa}-$ 
tients with $\mathrm{CHC}$ were treated with UDCA $600 \mathrm{mg} /$ day for 12 months, while 18 others did received placebo. A percutaneous liver biopsy was performed before and after 1 year of treatment. Histological analysis showed an improvement in the biliary features of the liver damage, although no significant modifications of the necroinflammatory scores were reported. ${ }^{33}$

Similar unsatisfactory results were reported in studies comparing the effects of IFN plus UDCA treatment versus IFN alone on the portal and periportal inflammation scores or on the Knodell score. ${ }^{24}$

Although no data exists on the ability of UDCA to modify the natural history of $\mathrm{CHC}$, the lack of efficacy on viral replication and the failure to improve liver histology have led to conclude that UDCA treatment per se is not able to slowdown or prevent the progression of chronic hepatitis into liver cirrhosis and HCC. ${ }^{24}$

At present, paramount advances in antiviral therapy introduce a new era of treatment for hepatitis $\mathrm{C}$ based on directly acting antiviral agents (oral protease inhibitors boceprevir and telaprevir), which are associated with a significant improvement in viral eradication rates in combination with pegylated IFN plus ribavirin. ${ }^{34}$ Further antiviral drugs, even more potent and effective, are under study. Therefore the chance to eradicate $\mathrm{HCV}$ infection is strikingly increased with respect to past decades, thus decreasing the need for an alternative therapy with BAs. ${ }^{34}$

Anyway, several patients with $\mathrm{CHC}$ are not candidates to new therapies, because of major contraindications, while others show non-response or relapses. In these cases, UDCA treatment might be taken into account with the aim to maintain low ALT levels, although it is not clear whether ALT normalization might modify the natural history of the disease. ${ }^{26}$

\section{Ursodeoxycholic acid and hepatitis B virus related chronic hepatitis}

Antiviral treatment of CHB is more effective than that of $\mathrm{CHC}$, therefore treatment with UDCA has been less used in this setting. ${ }^{35}$

The goal of antiviral therapy for CHB is to improve survival by preventing progression of the disease into cirrhosis and HCC. This goal can be achieved, if HBV replication can be suppressed in a sustained manner. ${ }^{36}$

Two different types of drugs can be administered to patients with CHB, IFN and nucleoside/nucleotide analogues (NAs). IFN is a cytokine with antiviral, antiproliferative, and immunomodulatory effects. It has shown to be effective in suppressing HBV replication and in inducing remission of liver disease. ${ }^{35,36}$ NAs act as inhibitors of the HBV polymerase activity. The more recent NAs (entecavir and tenofovir) show high potency, high genetic barrier, minimal side effects, low risk of viral resistance, oral administration, although they should be administered indefinitely. ${ }^{36}$

For these reasons, trials examining the use of UDCA in CHB are few and less recent than those in CHC. Available data confirms that UDCA decreases aminotransferase levels in patients with $\mathrm{CHB}$, similarly to CHC, although it is not be able to modify liver histology or decrease serum HBV DNA levels. ${ }^{24}$

No data exists on the ability of UDCA treatment to modify clinical outcome and to prevent progression to more severe liver damage. ${ }^{24}$

\section{Ursodeoxycholic acid and steatosis of the liver}

In western countries, liver steatosis is the main cause of an apparently unexplained increase of aminotransferases in the general population with a prevalence accounting for around $20-30 \% .{ }^{21}$ Liver fat derives from dietary free fatty acids (FFA), from liver FFA inflow, and from hepatic de novo lipogenesis. ${ }^{37}$

Steatosis might be distinguished in 2 different forms, alcoholic and non-alcoholic. The latter may be secondary to a variety of causes, such as overweight/ obesity, insulin resistance (IR), HCV, drugs, diabetes mellitus, disorders of lipid metabolism, rapid and severe weight loss, etc. ${ }^{37}$

NAFLD represents a spectrum of disorders characterized predominantly by macrovesicular steatosis occurring in individuals in the absence of significant alcohol consumption..$^{38}$ It is possible to distinguish a condition of simple fatty liver, where the only histologic finding is the presence of steatosis, from a state of $\mathrm{NASH}$, a potentially progressive hepatic disorder leading to end-stage liver disease, characterized by hepatocellular injury/inflammation with or without fibrosis. ${ }^{37}$ NAFLD is considered the hepatic manifestation of IR, and is therefore strongly associated with other clinical expressions of IR, such as metabolic syndrome and its features: obesity, type 2 diabetes, dyslipidemia and hypertension.$^{38}$ Although NAFLD is a rather benign condition, a fraction of these patients (20-30\%) with non-alcoholic fatty liver disease might develop more severe liver damage and liver cirrhosis. ${ }^{39}$

At present most hepatologists attempt to manage NASH using lifestyle changes to reverse the consequences of metabolic disease, such as weight reduction with or without exercise, as well as standard therapeutic interventions to control concomitant associated diseases, hyperlipidaemia, hypertension and type 2 diabetes. ${ }^{39}$

Pharmacological treatment of steatosis is still an unmet medical need. Due to the lack of specific and effective agents, several drugs have been studied: insulin-sensitizing agents, antioxidant therapy, vitamin E, betaine, pentoxifilline, probucol, omega-3 polyunsaturated fatty acids, sartans and lastly UDCA. ${ }^{40}$ 
In patients with fatty liver UDCA might prevent steatosis by protecting against mitochondrial injury, by inducing a plasma membrane stabilizing effect, and decreasing lipid peroxidation. Moreover, taurine-conjugated bile acids could inhibit the activity of some microsomal enzymes, which are induced by FFAs. UDCA inhibits the activation of Kupffer cells caused by toxic bile salts, thus acting as an antioxidant agent. ${ }^{41}$ Furthermore, UDCA increases hepatocyte levels of glutathione and thio-containing protein, therefore protecting hepatocytes against oxidative injury. ${ }^{42}$

UDCA may also reduce the expression of class II HLA inhibitors, the production of interleukin and interferon, thus acting as an immunomodulator. In addition, UDCA seems to reduce reactive oxygen species. ${ }^{43}$

Both UDCA and TUDCA have been tested, alone or in combination with other drugs, versus no intervention or in randomized controlled trials versus placebo. ${ }^{40}$

Biochemical response was assessed by serum activities of AST, ALT, alkaline phosphatase (ALP), GGT, and serum total bilirubin levels. UDCA or TUDCA treatments actually induce a decrease of AST and ALT serum levels, but there were no significant differences between patients treated with BAs and the control (placebo) group. Similarly, no significant decrease in GGT, ALP and bilirubin were seen. ${ }^{40}$

Conflicting results were reported as to ultrasound (US) response. The majority of trials did not show significant improvement of US features of steatosis after UDCA treatment, ${ }^{40}$ only one study reported a slight decrease in the US steatosis score. ${ }^{44}$ Therefore, available data allow to exclude any radiological benefit of UDCA versus placebo/no intervention in patients with fatty liver.

The major clinical outcome of such treatment should be the possible improvement of histological liver damage. Only a few studies have addressed this issue, failing to show significant modifications of degree of steatosis, inflammation, or fibrosis among treated patients. Lindor et $a l .{ }^{43}$ studied the efficacy of UDCA in 166 patients with biopsy-proven NASH. End-points included changes in liver test results and liver histology at 2 years of therapy. Unfortunately, also this study failed to show any biochemical or histological benefit of UDCA in patients with NASH.

More recently, Ratziu et al. ${ }^{41}$ investigated the efficacy and safety of high-dose UDCA (HD-UDCA, 28$35 \mathrm{mg} / \mathrm{kg}$ per day) in a 12-month, randomized, double-blind, placebo-controlled multicenter trial enrolling 126 patients with biopsy-proven NASH and elevated ALT levels. The primary study end-point was a reduction in ALT levels from baseline in patients treated with HD-UDCA compared with placebo. Secondary study end-points were the proportion of patients with ALT normalization, a relative reduction in the scores of serum markers of fibrosis and hepatic inflammation, and safety and tolerability.

In this study, HD-UDCA significantly reduced mean ALT levels ( $-28.3 \%$ from baseline after 12 months compared with $-1.6 \%$ with placebo, $\mathrm{P}<0.001$ ). At the end of the trial, ALT levels normalized in $24.5 \%$ of patients treated with HD-UDCA and in $4.8 \%$ of patients who received placebo $(\mathrm{P}=0.003)$. Both results were not accounted for by changes in weight during the trial.

HD-UDCA significantly reduced the serum fibrosis marker compared with placebo. Furthermore, this BA also significantly improved markers of glycemic control and insulin resistance. ${ }^{41}$ The results of this study indicate that probably UDCA should be administered in a dosage higher than that reported in previous trials.

\section{Conclusions}

Although UDCA is extensively prescribed in clinical practice for the treatment of patients with hypertransaminasemia of various origins, its use is hampered by the fact that it is not clear whether biochemical remission seen during UDCA treatment might be associated with histological improvement and a favorable modification of the disease progression. Moreover, it should be underlined that in our country UDCA treatment for non-cholestatic chronic liver disease is considered off-label and therefore it is not refunded by our National Health System.

On the other side, absence of evidence does not always mean absence of effect. ${ }^{40}$ Furthermore, it should be considered that UDCA is a rather safe and relatively inexpensive drug. ${ }^{26}$ The decrease of liver biochemistry during treatment often reduces patient anxiety, thus improving quality of life and mental health status (Elmo MG, unpublished data, 2012).

Lastly, although it is not clear whether ALT levels actually correlate with the severity of liver damage and disease progression, particularly in $\mathrm{CHC},{ }^{45}$ we cannot rule out the possibility that persistent ALT improvement or even normalization might favorably modify the clinical outcome and the long-term history of chronic liver diseases.

In conclusion, further prospective studies using higher doses of UDCA and/or longer treatment periods are needed. Patients with $\mathrm{CH}$ should be clearly informed that this therapy might merely have cosmetic effects on the liver biochemistry.

\section{References}

1. Hofmann AL. Bile acids: trying to understand their chemistry and biology with the hope of helping patients. Hepatology 2009;49:1403-18.

2. Lazaridis KN, Gores GJ, Lindor KD. Ursodeoxycholic 
acid. Mechanisms of action and clinical use in hepatobiliary disorders. J Hepatol 2001;35:134-46.

3.Pauli-Magnus C, Stieger B, Meier Y, et al. Enterohepatic transport of bile acids and genetics of cholestasis. J Hepatol 2005;43:342-57.

4. Chiang JYL. Regulation of bile acid synthesis: pathways, nuclear receptors, and mechanisms. J Hepatol 2004;40:539-51.

5. Russel DW. The enzymes, regulation and genetics of bile acid synthesis. Ann Rev Biochem 2003;72:137-74.

6. Meier PG, Stieger B. Bile salt transporters. News Physiol Sci 2000;15:89-93.

7. Hofmann AL. Gallstone disease: physicochemical research sheds new light on an old disease and points the way to medical therapy (commentary). J Hepatol 2004;41:195-200.

8. Admirand WH, Small DM. The physicochemical basis of cholesterol gallstones formation in man. J Clin Invest 1968;47:1043-52.

9. Danzinger RG, Hofmann AF, Schoenfield LJ, Thistle JL. Dissolution of cholesterol gallstones by chenodeoxycholic acid. N Engl J Med 1972;286:1-8.

10. Nakagawa S, Makino I, Ishazaki T, Dohi I. Dissolution of cholesterol gallstones by ursodeoxycholic acid. Lancet 1977;2:367-9.

11. Hofmann AF. Pharmacology of ursodeoxycholic acid, an enterohepatic drug. Scand J Gastroenterol 1994;29:1-15.

12. Bachrach WH, Hofmann AF. Ursodeoxycholic acid in the treatment of cholesterol cholelithiasis. Dig Dis Sci 1982;27:737-61.

13. Poupon R, Chretien Y, Poupon RE, et al. Is ursodeoxycholic acid an effective treatment for primary biliary cirrhosis ? Lancet 1987;1:834-6.

14. Poupon RE, Balkau B, Eschwege E, et al. A multicenter, controlled trial of ursodiol for the treatment of primary biliary cirrhosis. N Engl J Med 1991;324:1548-54.

15. Poupon RE, Poupon R, Balkau B, for the UDCA-PBC study group. Ursodiol for the long-term treatment of primary biliary cirrhosis. N Engl J Med 1994;330: 1342-7.

16. Lindor KD, Therneau TM, Jorgensen RA, et al. Effects of ursodeoxycholic acid on survival in patients with primary biliary cirrhosis. Gastroenterology 1996;110:1515-8.

17. Kowdley KV. Ursodeoxycholic acid therapy in hepatobiliary disease. Am J Med 2000;108:481-6.

18. Goulis J, Leandro G, Burroughs AK. Randomised controlled trials of ursodeoxycholic acid therapy for primary biliary cirrhosis: a meta-analysis. Lancet 1999;354: 1053-60.

19. Rudic JS, Poropat G, Krstic MN, et al. Ursodeoxycholic acid for primary biliary cirrhosis. Cochrane Database Syst Rev 2012;12:CD000551.

20. Green RM, Flamm S. AGA technical review on the evaluation of liver chemistry tests. Gastroenterology 2002;123:1367-84.

21. Bellentan S, Tiribelli C. The spectrum of liver disease in the general population. Lessons from the Dyonisos study. J Hepatol 2001;35: 531-7.

22. Poynard T, Yuen MF, Ratziu V, Lai CL. Viral hepatitis C: review. Lancet 2003;362:2095-100.

23. Puoti C, Pannullo A, Annovazzi G, et al. Ursodeoxycholic acid and chronic hepatitis $\mathrm{C}$ infection. Lancet 1993;341:1413-4.
24. Chen W, Liu JP, Gluud C. Bile acids for viral hepatitis. Cochrane Database Syst Rev 2007;(4):CD003181.

25. Takano S, Nakamura K, Yokosuka O, et al. A multicenter randomized controlled dose study of ursodeoxycholic acid for chronic hepatitis C. Hepatology 1994; 20:558-64.

26. Puoti C, Magrini A, Filippi T, et al. Effects of ursodeoxycholic acid on serum liver enzymes in patients with hepatitis $\mathrm{C}$ virus-related chronic liver disease. Eur J Gastroenterol Hepatol 1995;7:151-4.

27. Scotto G. The ursodeoxycholic acid for the treatment of HCV infections. Inf Med 1997;3:168-73.

28. Crosignani A, Battezzati PM, Cestari C, et al. .Tauroursodeoxycholic acid for the treatment of chronic hepatitis: a multicentre dose-response study. Hepatol Res 1998;13:10-9.

29. Belloni G, Ferrari L, Balduzzi C, et al. Effect of tauroursodeoxycholic acid on ALT, GGT and viral load in patients with hepatitis $\mathrm{C}$ virus-related chronic hepatitis with reference to the genotype. Ital J Gastroenterol Hepatol 1999;31:427.

30. Angelico M, Gandin C, Pescarmona E, et al. Recombinant interferon alpha and ursodeoxycholic acid versus interferon alpha alone in the treatment of chronic hepatitis C: a randomized clinical trial with long-term follow-up. Am J Gastroenterol 1995;90:263-9.

31. Ohiwa T, Katagiri K, Hoshino M, et al. Tauroursodeoxycholate and tauro-beta-muricholate exert cytoprotection by reducing intrahepatocyte taurochenodeoxycholate content. Hepatology 1993;17:470-6.

32. Nojiri S, Nakao H, Sugauchi F, et al. Effect of ursodeoxycholic acid on serum liver enzymes and bile acid metabolism in chronic active hepatitis $\mathrm{C}$ virus infection. Hepatol Res 2009;39:21-30.

33. Attili AF, Rusticali A, Varriale M, et al. The effect of ursodeoxycholic acid on serum enzymes and liver histology in patients with chronic active hepatitis. A 12-month double-blind, placebo-controlled trial. J Hepatol 1994;20:315-20.

34. Asselah T. A revolution in HCV treatment with directacting antivirals: From non-response to eradication. J Hepatol 2012;57:455-7.

35. Puoti C. How to manage HBeAg-negative chronic HBV infection with normal alanine aminotransferase levels in clinical practice? Eur J Intern Med 2013;24:100-3.

36. European Association for the Study of the Liver (EASL). EASL clinical practice guidelines: management of chronic hepatitis B virus infection. J Hepatol 2012;57:167-85.

37. Sanyal AJ. AGA Technical review on non-alcoholic fatty liver disease. Gastroenterology 2002;123:1705-25.

38. Vuppalanchi R, Chalasani N. Non-alcoholic fatty liver disease and non-alcoholic steatohepatitis: selected practical issues in their evaluation and management. Hepatology 2009;49:306-17.

39. Comar KM, Sterling RK. Drug therapy for non-alcoholic fatty liver disease. Aliment Pharmacol Ther 2006;23:207-15.

40. Orlando R, Azzalini L, Orando S, Lirussi F. Bile acids for non-alcoholic fatty liver disease and/or steatohepatitis. Cochrane Database Syst Rev 2007;(1):CD005160.

41. Ratziu V, de Ledinghen V, Fréderic Oberti F, et al. A randomized controlled trial of high-dose ursodesoxycholic 
acid for non-alcoholic steatohepatitis. J Hepatol 2011; 54:1011-9.

42. Bellentani S, Chao YC, Ferretti I, et al. Chronic administration of ursodeoxycholic and tauroursodeoxycholic acid changes microsomal membrane lipid content and fatty acid compositions in rats. Biochem Biophys Res Commun 1996;220:479-83.

43. Lindor KD, Kowdley KV, Heathcote EJ, et al. Ursodeoxycholic acid for treatment of non-alcoholic steatohepatitis: results of a randomized trial. Hepatology 2004;39:770-8.

44. Mèndez-Sanchez N, Gonzalez V, Chavez-Tapia N, et al. Weight reduction and ursodeoxycholic acid in subjects with non-alcoholic fatty liver disease. A double-blind, placebo controlled trial. Ann Hepatol 2004;3:108-12.

45. Puoti C. HCV carriers with persistently normal aminotransferase levels: normal does not always mean healthy. J Hepatol 2003;38:529-32. 